Article

\title{
Fusion of Multiple Pyroelectric Characteristics for Human Body Identification
}

\section{Wanchun Zhou, Ji Xiong, Fangmin Li *, Na Jiang and Ning Zhao}

Key Laboratory of Fiber Optical Sensing Technology and Information Processing, Ministry of Education, Hubei Key Laboratory of Broadband Wireless Communication and Sensor Network, Wuhan University of Technology, Wuhan, Hubei 430070, China; E-Mail: wanchun_zhou@126.com (W.Z.); whutinfo@whut.edu.cn (J.X.); WHUTjiangna@163.com (N.J.); zhaoning@whut.edu.cn (N.Z.)

* Author to whom correspondence should be addressed; E-Mail: lifangmin@whut.edu.cn; Tel.: +86-027-8729-0335; Fax: +86-027-8729-0216.

External Editor: Marc Sevaux

Received: 13 October 2014; in revised form: 8 December 2014 / Accepted: 10 December 2014 / Published: 18 December 2014

\begin{abstract}
Due to instability and poor identification ability of single pyroelectric infrared (PIR) detector for human target identification, this paper proposes a new approach to fuse the information collected from multiple PIR sensors for human identification. Firstly, Fast Fourier Transform (FFT), Short Time Fourier Transform (STFT), Wavelet Transform (WT) and Wavelet Packet Transform (WPT) are adopted to extract features of the human body, which can be achieved by single PIR sensor. Then, we apply Principal Component Analysis (PCA) and Support Vector Machine (SVM) to reduce the characteristic dimensions and to classify the human targets, respectively. Finally, Fuzzy Comprehensive Evaluation (FCE) is utilized to fuse recognition results from multiple PIR sensors to finalize human identification. The pyroelectric characteristics under scenarios with different people and/or different paths are analyzed by various experiments, and the recognition results with/without fusion procedure are also shown and compared. The experimental results demonstrate our scheme has improved efficiency for human identification.
\end{abstract}

Keywords: pyroelectric infrared (PIR) sensor; feature extraction; principal component analysis (PCA); support vector machine (SVM); fuzzy comprehensive evaluation method (FCEM) 


\section{Introduction}

Biometric identification technology mainly consists of two kinds of identification techniques based on physical characteristics and behavioral characteristics, respectively. Physical characteristics usually include face, fingerprint, retina, etc.; and, behavioral characteristics include signatures, voice, gait, and so forth. Since gait recognition has many advantages in the long-distance identification, it has drawn many researchers' attentions and is becoming an emerging biometric identification research topic. Besides, traditional video systems have been applied to many recognition scenes [1,2]. Such systems, however, primarily identify persons through facial features which are greatly restricted by many external factors such as lighting, angle and clothes. Additionally, they usually have high computational overhead and require huge data throughput.

In recent years, with the rapid development of the sensor technology, distributed inference and learning technology, human behavioral information can be detected and measured by passive (e.g., thermal or pressure) or active sensors (e.g., ultrasound or laser); and spatially distributed sensors with decent computation and communication capabilities can work together to achieve complex tasks. Therefore, researchers have attempted to combine their merits to develop the distributed sensor networks based on pyroelectric infrared sensors [3,4]. In order to identify human objects, the pyroelectric infrared sensors have many advantages as follows:

- Reductions both in the number of measurements and in sampling frequency for human motion state estimation.

- Reductions in hardware cost, power consumption, privacy, infringement, computational complexity, communication overhead, and networking data throughput.

- Reductions in system deployment duration, limitations upon applications or application location restrictions (e.g., long range or crowded scene).

- Its performance is independent of illumination and has strong robustness to the color change of background.

- Its sensitivity range of angular rate is about $0.1 \mathrm{r} / \mathrm{s}$ to $3 \mathrm{r} / \mathrm{s}[5,6]$, which can cover the most human walking speeds at around 2-10 m. It can obtain better field of view (FOV) combined with low price Fresnel lens array. Thus, compared with the traditional video systems, distributed wireless pyroelectric sensor networks can provide better spatial coverage and reduce the deployment duration and deployment location restrictions.

Now, distributed pyroelectric infrared network equipped with plenty of sensors can collect various information about changes in the users' states and surroundings. In particular, such systems interpret sensor data as a reflection of user behavior, and thus can build a rich model of the user context. Accordingly, such activity knowledge can help systems adapt their services and computational resources to the user context, e.g., controlling light, or adjusting heating, etc.

This paper uses the distributed pyroelectric infrared sensors as the source of information collection in specific environment. Referring recent research [7], the system in this paper consists of four sensor modules, data processing node, wireless gateway and a host computer. Here, each sensor module contains a pyroelectirc infrared sensor which is covered by several Fresnel lens and a signal modulation mask. Once a human target moves into the sensing area of the sensor, the infrared radiation of the human 
target can be captured and transformed into electrical signal which can be transmitted to the host computer to be further processed through the wireless gateway after preliminary noise reduction and data compression.

However, before using a distributed wireless pyroelectric infrared sensor network in the identification procedure of motion human target, much attention should be paid to the following items:

- Different Fresnel lens and signal modulation mask can obtain more pyroelectric infrared information of the human target.

- The four sensors are installed with different heights, which can collect different pyroelectric infrared information from corresponding parts of the human body.

- The effective data is fused by multiple channel signals which are collected from the four sensor modules.

- Extracting different pyroelectric infrared features of the human target by different algorithms can help establish different target identification model databases.

In this paper, the major goal of the work is to develop a novel wireless distributed pyroelectric sensor system which can precisely identify human targets in a confined area. With the increasing distance between the pyroelectric sensor and the human target, the target recognition rate of a single sensor module is showed in a downward trend. Thus, a novel fusion algorithm to fuse the data of pyroelectric sensors with different heights is proposed to improve recognition rate. Firstly, Fast Fourier transform (FFT), Short Time Fourier Transform (STFT), Wavelet Transform (WT) and Wavelet Packet Transform (WPT) are used to extract the pyroelectric features of the human body, which is detected by Single Pyroelectric Infrared (PIR) sensor. Then, the dimensionality of pyroelectric features of the human target is reduced through the Principal Component Analysis (PCA). Additionally, the preliminary recognition rate of single sensor module is obtained by using the Support Vector Machine (SVM) algorithm. Finally, the Fuzzy Comprehensive Evaluation method (FCE) is applied to fuse the preliminary recognition rate of the four PIR sensor modules, and then the final recognition result can be obtained. The experiments proved that the proposed scheme has stronger robustness on the human height and the detection distance between the human target and the pyroeletric sensor.

The remainder of the paper is organized as follows. The related work is illustrated in Section 2. Section 3 introduces sensor modules and the deployment structure. Section 4 presents the proposed human target recognition system, and a novel recognition strategy is presented in Section 5. The experimental results with the various collected data are presented and analyzed in Section 6. Section 7 concludes the paper.

\section{Related Work}

The PIR sensors are widely used in surveillance systems and automatic light switching systems as simple but reliable triggers [8,9]. They also have shown promising capabilities as low-cost camera enhancers in video surveillance systems. The work of Rajgarhia et al. [10] uses PIR sensors in conjunction with cameras to address privacy issues. PIR sensors are deployed in private rooms while cameras in public areas. Tao et al. [11] presented a person localization algorithm using an infrared ceiling sensor network for providing various personalized services in an office environment, which 
demonstrates the benefits of reducing camera deployment by introducing PIR sensors. In [12], a kind of platform for home surveillance is proposed. The platform includes an ARM processor together with a Web camera and a PIR sensor. The PIR sensor triggers the Web camera in presence of an intruder in order to capture and send a snapshot to a remote server.

Other works present different approaches to perform human body tracking and identifying using only PIR sensors. As for tracking, Hao et al. [13] presented a human tracking system using a node with eight PIR detectors and Fresnel lens arrays arranged around a circle; and the system can be used to track single human target by detecting its angular displacement while moving. Similarly, the authors also introduced multiple human tracking and identification with wireless distributed PIR sensor systems.

Shankar et al. [14] developed a human tracking system using a low-cost sensor cluster consisting of PIR sensors and Fresnel lens arrays to implement the desired spatial segmentations. They analyzed the response characteristics of the sensor cluster, and extracted velocity and the direction of motion over large areas of more than $12 \mathrm{~m}$. Kim et al. [15] presented a location-aware system, which uses an array of PIR sensors. In such system, the PIR sensors, on the ceiling with overlapping detective areas of adjacent sensors, can detect the presence of a resident. However, this system cannot work when there are multiple targets or it is deployed at vacant zones. In Luo et al [16], four sensor modules, each of which consists of five PIR detectors, are mounted on the ceiling of a monitor field to fix the position of a moving human target. In addition, Kalman filter is adopted to improve the tracking accuracy. Lu et al. [17] developed a compressive multi-target tracking system used in binary PIR sensor networks. Each sensor node can detect multiple small regions instead of a large one in order to achieve high sensing resolution and efficiency.

In terms of recognition, Fang et al. [18] presented a human identification system using a PIR sensor whose visibility is modulated by a Fresnel lens array and principal components regression method. They also presented a method for identifying subjects walking randomly by using PIR sensors with modulated visibilities and hidden Markov models [18,19]. Zhou et al. [20] developed a wireless distributed pyroelectric sensor system to recognize different walkers' gait features. Sun et al. [21] built a distributed binary pyroelectric sensor network (PSN) for the purpose of multi-walker recognition and tracking. They accurately extract context features from a hybrid, binary, multi-walker sensor data stream to identify and track multi-walker and achieve good results, in the four tested scenarios with $100 \%$ success rate. $\mathrm{Hu}$ et al. [22] used binary principle component analysis (B-PCA) to interpret the relationship between observed sensor data and hidden context patterns, and conducted comprehensive experiments from real sensor data to verify the context detection accuracy. Sun et al. [23] proposed a statistical subspace representation model called probabilistic nonnegative matrix factorization (PNMF) to seek the scenario patterns rather than the object characteristics. They further prove that their PNMF model is a generic model for nonnegative matrix factorization (NMF) based algorithms [24]. Experimental result demonstrate the advantages of their proposed method.

The advantages of the wireless distributed sensor system include the convenient deployment of multiple sensor nodes for collecting measurements from multiple perspectives. By using multiple sensor nodes, the human motion feature can be accurately captured and utilized for the higher-security applications where walker verification or open-set identification is required. A typical biometric system consists of three modules: feature extraction, matching, and decision. Feature extraction is used to describe the most important information of the sensory data (samples). Matching modules compare 
feature with templates in the database and output a score to the decision module. Therefore, the information fusion of multiple pyroelectric sensor nodes for thermal gait biometrics can happen at four different levels: sample, feature, score, and decision.

In this paper, a novel application is proposed that multiple PIR sensor modules are utilized to collect pyroelectic data of different parts of the human body. Additionally, various algorithms are applied to extract thermal infrared features of different parts of the human body. Then, the decision-making algorithm is used to fuse multiple recognition results of the system to obtain final human identification. Through multiple experiments, we analyzed the pyroelectric characteristics of different human targets and different paths. Also, we compared the recognition results with and without fusion procedure, and demonstrated that the proposed approach combined with the characteristic extraction, fusion and recognition method can effectively remedy recognition deficient of the system with single PIR sensor.

\section{Sensor Modules and Deployment}

Referring to the system that is set up by Qi Hao in [12], we build a distributed pyroeletric infrared sensor network, composed of several sensor nodes, a wireless gateway and a host computer, to detect and identify motion human target.

\subsection{PIR Sensor Module}

Because of the relatively stable performance, the $\mathrm{LiTaO}_{3}$ film pyroelectric infrared sensor is chosen as the detecting node in the system. Due to the lower receiving sensitivity of the sensor itself, each signal sensor node is covered by a Fresnel lens as shown in Figure 1. It cannot only focus the infrared heat to the sensor node, but also can increase the angle and detectable distance. It was proved in some experiments that the effective detectable range can vary from 2 to 12 or $14 \mathrm{~m}$. Based on the parameters and characteristics of the D205B sensor as shown in Table 1, the PIR sensor module is designed as shown in Figure 1.

As shown in Figure 2, the original thermal infrared signal of human target can be translated into analog signal through three steps. Firstly, the original human thermal infrared signal is focused on the pyroelectric sensor by using Fresnel lens. Then, the infrared signal is translated into weak electrical signal by PIR sensor. Finally, the analog signal can be obtained after the processing unit.

Figure 1. A pyroelectric infrared sensor module is covered by Fresnel lens and signal modulation mask.

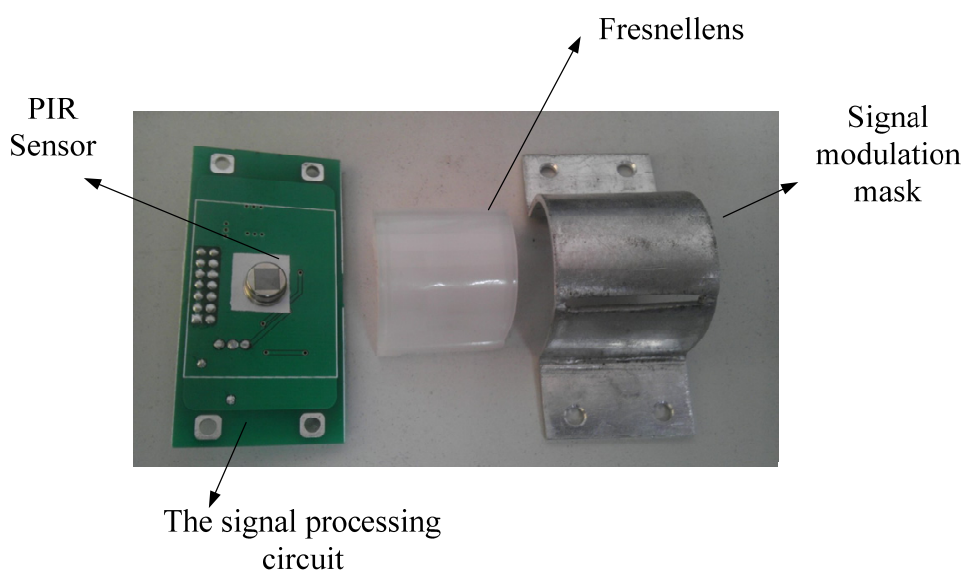


Table 1. The Parameters of D205B Pyroelectric Infrared Sensor.

\begin{tabular}{cc}
\hline Parameters & Value \\
\hline IR Receiving Electrode & $0.7 \times 2.4 \mathrm{~mm}, 4$ elements \\
Sensitivity & $\geq 4300 \mathrm{~V} / \mathrm{W}$ \\
Detectivity (D*) & $1.6 \times 108 \mathrm{~cm}(\mathrm{~Hz})^{1 / 2} / \mathrm{W}$ \\
Supply Voltage & $3-15 \mathrm{~V}$ \\
Operating Temp & $-30-70{ }^{\circ} \mathrm{C}$ \\
Offset Voltage & $0.3-1.2 \mathrm{~V}$ \\
FOV & $150^{\circ}$ \\
\hline
\end{tabular}

Figure 2. Human thermal infrared signal processing in sensor module.

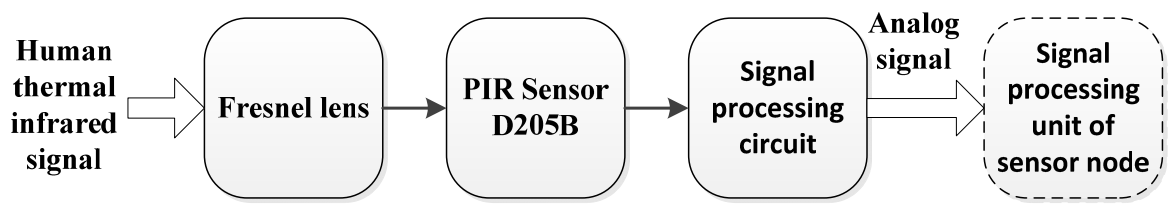

\subsection{PIR Sensor Node}

The PIR sensor node consists of four sensing units (PIR sensor module), a processing unit, and a communication subsystem. The sensing unit is usually composed of PIR sensor, Amplifier and Actuator. The analog original signal captured by sensor module is primarily amplified by amplifier, and then it is converted to digital signal by the Analog to Digital Converter (ADC) module. In the processing unit, the STM32 CPU has embedded $256 \mathrm{kB}$ of flash memory and $48 \mathrm{kB}$ of RAM for program and data. Furthermore, all the coordinating sensing and communication tasks are executed by this 32-bit CPU at $72 \mathrm{MHz}$. The memory sub-unit can store sensing data in a period of time. The communication subsystem composed of a transceiver sub-unit and a processing circuit connects the device to the network.

The processed signal is sent to the wireless gateway by communication subsystem on the basis of Zigbee protocol in the specified interval. Moreover, the whole system is powered by a power unit that can be supported by an energy scavenging unit such as solar cells. The physical device is shown in Figure 3.

Figure 3. The composition of pyroelectric infrared (PIR) senor node.

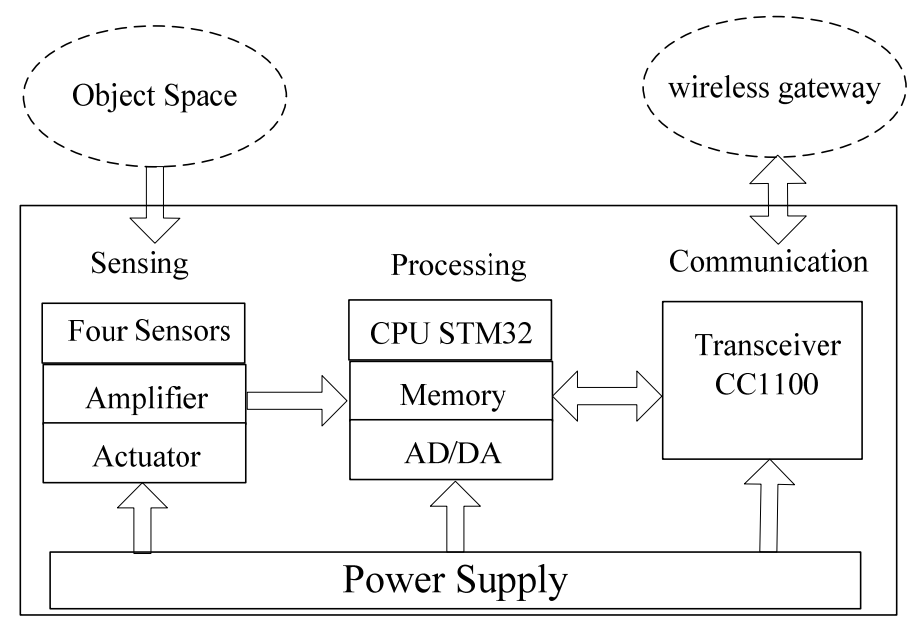




\subsection{Gateway Module}

Gateway module is composed of four wireless modules units, a STM32 processor and a gateway unit. The signals of the human target, which are detected by each sensor node, were all transmitted to the wireless module CC1100 in a specified interval. Because four wireless modules have four kinds of channels and they connect with the STM32 processor by General Purpose Input Output (GPIO), the STM32 processor can independently process the different data from sensor nodes, and it can also complete wireless data acquisition at the same time. Moreover, sensed data will be transmitted to a host computer through Ethernet interface for further processing and display. The whole structure and physical photo of the wireless gateway module are shown in Figures 4 and 5, respectively. Besides, the whole system is powered by a power unit.

Figure 4. The composition module of wireless gateway.

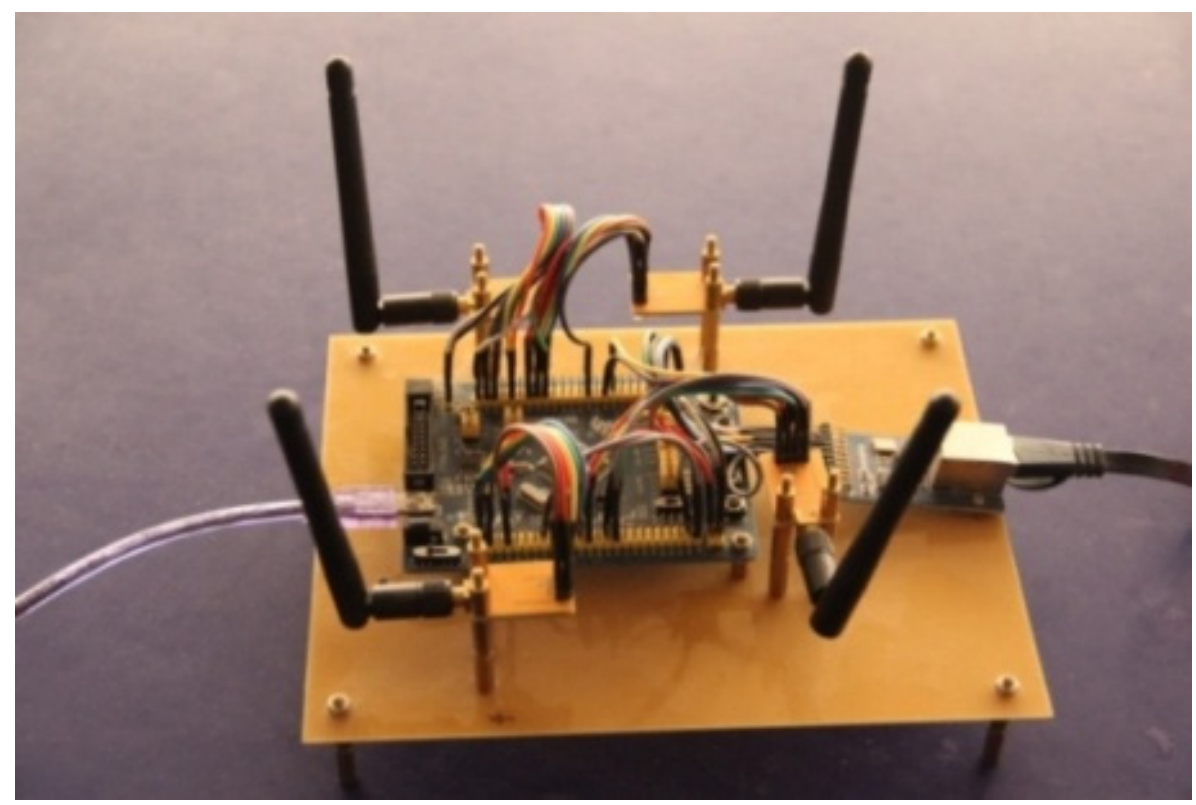

Figure 5. The wireless gateway module.

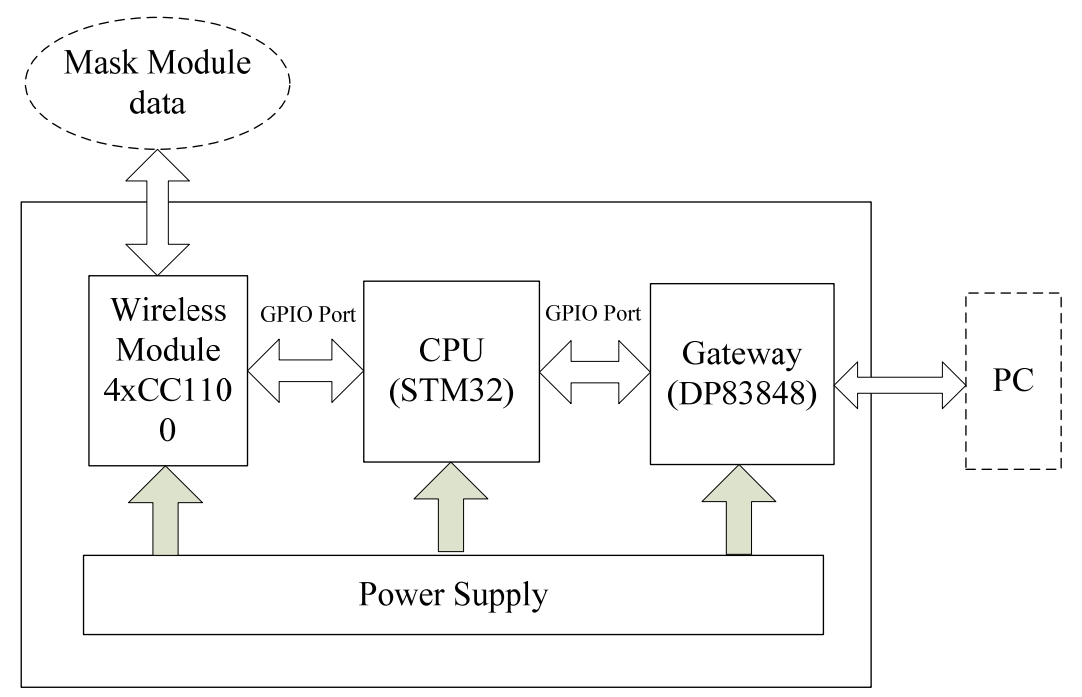




\section{Target Recognition System}

\subsection{System Architecture}

Target recognition system consists of two parts, namely data acquisition part and data processing part. Data acquisition part is divided into three units: sensing unit, wireless unit, PC monitor terminal unit. When the target enters into the detection area, the infrared signals are emitted by the human body can converge to PIR sensors through the aluminum mask and Fresnel lens, and then they are turned into an electrical signal. After that, the electrical signal is filtered and amplified. It is converted into digital signal by the A/D converter circuit. Through the point to point data transmission between Nrf24L01 wireless unit and wireless unit of gateway, data will be transferred to the PC software by the gateway based on the TCP/IP protocol. In this paper, the Qt4.7.4 software platform is used to develop the PC software primarily designed for data reading, storing and waveform drawing. The process of data collection is shown in Figure 6.

Figure 6. Data acquisition flow chart.

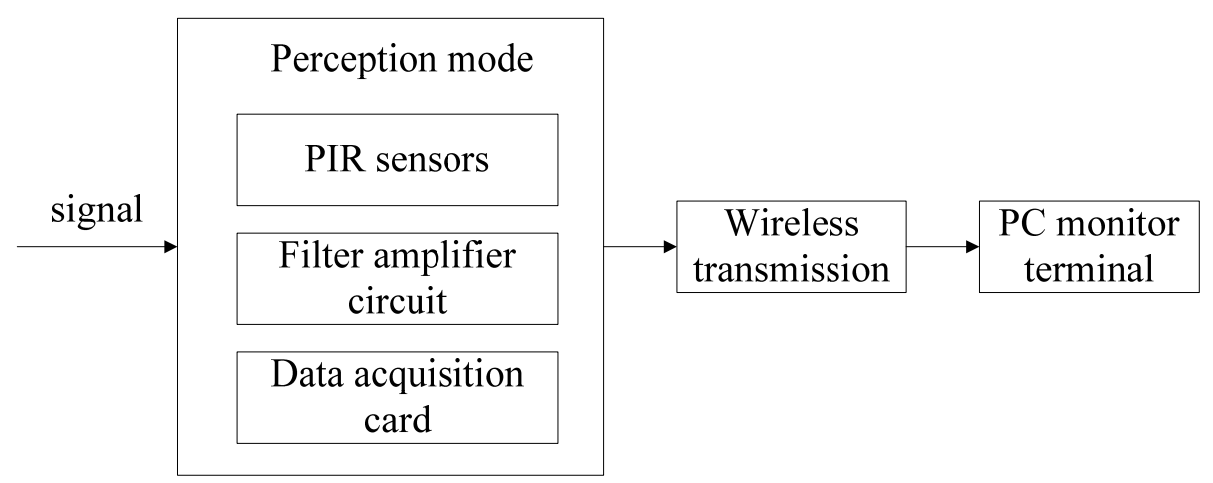

The data processing part is mainly composed of three parts: feature extraction, feature matching and feature determination. In the feature extraction unit, this paper adopts FFT, STFT analysis method, WT and WPT, which are respectively used to extract the characteristics of sample data. Then, we can use SVM algorithm to classify and match the sample data in order to get a preliminary recognition rate. Furthermore, we can also analyze the effect on recognition rate caused by different installation height of the sensor and the distance between the human target and the PIR sensor node. Based on these factors, the FCEM is used to fuse the recognition data from four different sensors for the final recognition results.

\subsection{Experimental Program}

The test model is shown in Figure 7. The size of the area is $6 \mathrm{~m} \times 6 \mathrm{~m}$. The sensors heights are set to 0.4, 0.8, 1.2 and $1.6 \mathrm{~m}$. The design of different height sensors aims at the research of features. Among these heights, $0.4,0.8,1.2$ and $1.6 \mathrm{~m}$ correspond to the part of the human knee, the human hand swing, the human chest and the human head, respectively. In this experiment, the test objects are 10 healthy young students aged at 23-24 years old. The heights of different human bodies are shown in Table 2. The test objects were required to walk along the predefined six lines marked as A, B, C, D, E and F at normal speeds with 10 times per line. 
The purpose of this experiment is to study the impact of two factors on the recognition rate, i.e., the height of sensor location and the vertical distance between the PIR node and the walking path. Furthermore, the decision fusion based on FCEM is proposed in order to improve the system recognition results.

Table 2. Attribute Features of Experiment Object.

\begin{tabular}{cccc}
\hline Object & Sexuality & Height & Step distance \\
\hline $\mathrm{a}$ & male & $175 \mathrm{~cm}$ & $50 \mathrm{~cm}$ \\
$\mathrm{~b}$ & male & $167 \mathrm{~cm}$ & $40 \mathrm{~cm}$ \\
$\mathrm{c}$ & female & $160 \mathrm{~cm}$ & $40 \mathrm{~cm}$ \\
$\mathrm{~d}$ & male & $182 \mathrm{~cm}$ & $60 \mathrm{~cm}$ \\
$\mathrm{e}$ & male & $172 \mathrm{~cm}$ & $50 \mathrm{~cm}$ \\
$\mathrm{f}$ & female & $158 \mathrm{~cm}$ & $37 \mathrm{~cm}$ \\
$\mathrm{~g}$ & female & $162 \mathrm{~cm}$ & $40 \mathrm{~cm}$ \\
$\mathrm{~h}$ & male & $179 \mathrm{~cm}$ & $56 \mathrm{~cm}$ \\
$\mathrm{i}$ & male & $173 \mathrm{~cm}$ & $50 \mathrm{~cm}$ \\
$\mathrm{j}$ & female & $171 \mathrm{~cm}$ & $52 \mathrm{~cm}$ \\
\hline
\end{tabular}

Figure 7. Experimental scene.

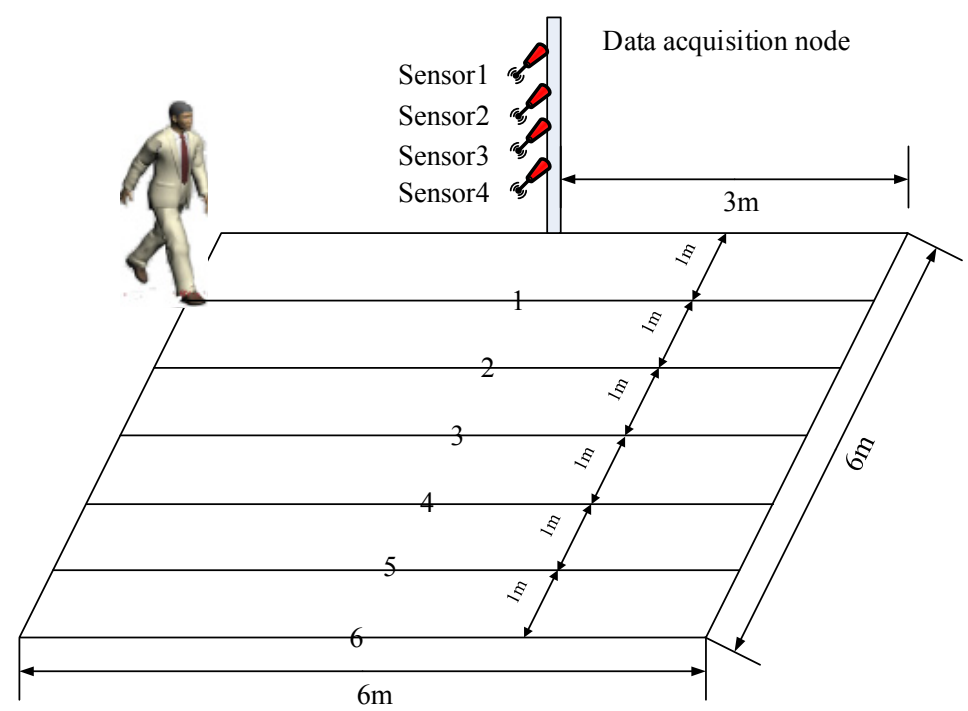

\section{Algorithm Descriptions}

The Algorithm can be divided into three modules: feature extraction module, SVM classification module and FCEM fusion module [25,26]. The architecture is shown in Figure 8. In this paper, firstly, the characteristics of original signal are extracted. Then, through SVM classifier, the signal is further processed to get the recognition result of each sensor. Finally, the FCEM is used to fuse the preliminary recognition result of the four different sensors to obtain the final results.

In the feature extraction section, four different algorithms are used, namely FFT, STFT, WT, WPT. It aims to verify and compare the recognition results of different sensors under various feature extraction algorithms.

In the data fusion section, we can get the recognition result of each sensor through the data analysis. However, the results, especially the effects of distance, are not often so good. Therefore, this paper 
adopts FCEM to fuse the classification results from each sensor and then get the final recognition result. The structure of the proposed algorithm is shown in Figure 8.

Figure 8. Algorithms Chart.

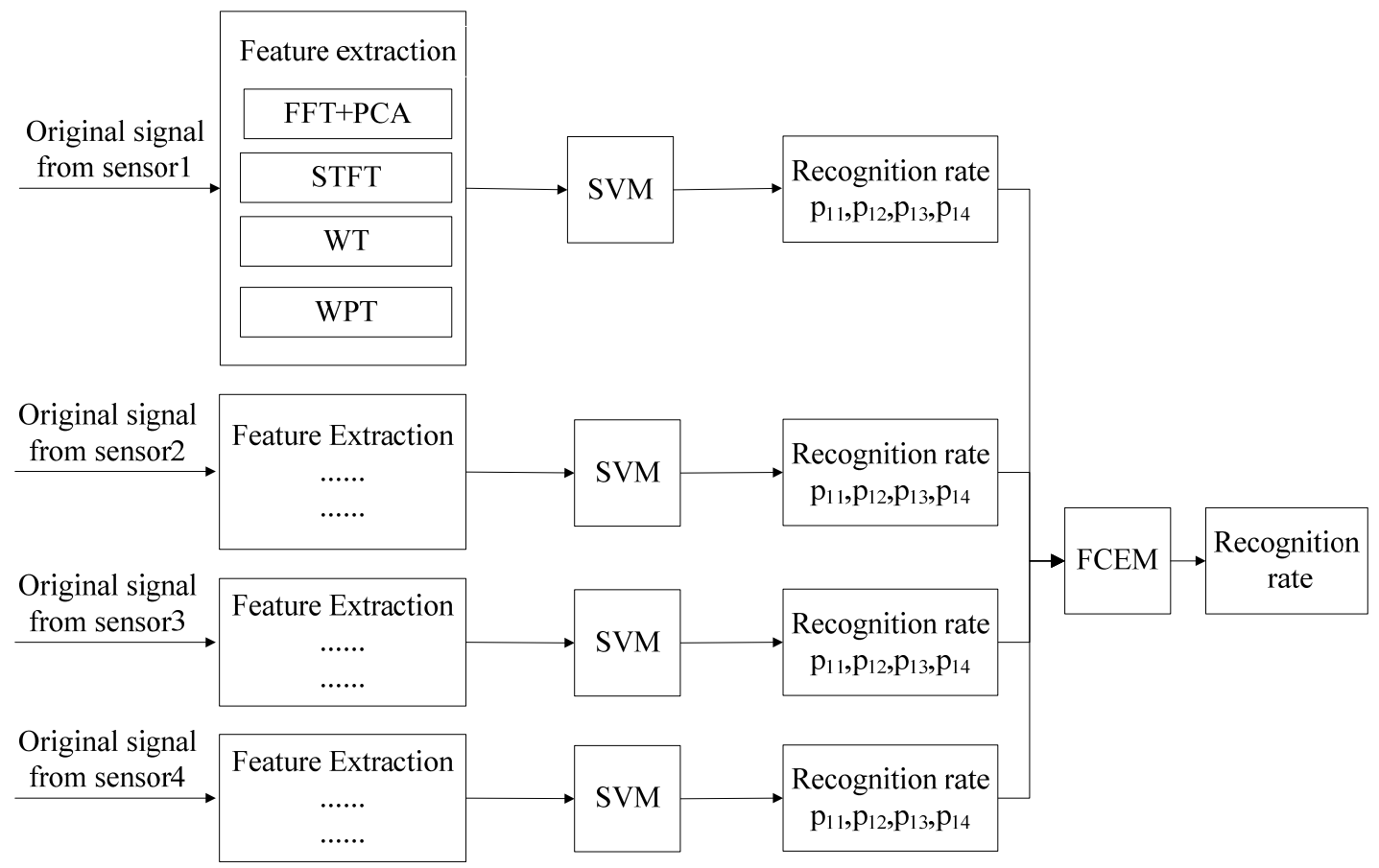

\subsection{Feature Extraction}

Because the feature extraction explicitly affects the final recognition result, this paper uses a variety of algorithms for feature extraction.

\subsubsection{FFT + PCA (Fast Fourier Transform and Principal Component Analysis)}

The sample signals obtain the amplitude spectrum through the Fast Fourier Transform. High spectrum dimension can reduce classifier performance. The principal component analysis (PCA) method is adopted to reduce the dimension of spectrum data.

$X$ is the $N$ observations of $p$ variable. $X=\left[x_{1}, x_{2}, x_{3}, \ldots, x_{n}\right]^{\mathrm{T}}, x_{1}: n$ is a row vector of $p$ dimension which means the spectrum characteristics of each sample, where $p$ is the number of the spectrum points. PCA algorithm are briefly described as below [27]:

- Standardize the observation matrix $X$ to obtain matrix $Y$;

- Calculate $Z$ which is the covariance of matrix $Y$;

Calculate the covariance matrix eigenvalue and eigenvector of $Z$, and order them from large to small order as $\lambda_{1} \geq \lambda_{2} \geq \lambda_{3} \geq \ldots \geq \lambda_{p}$. The corresponding eigenvectors are $U_{1}, U_{2}, U_{3}, \ldots, U_{p}$, and covariance matrix $\mathrm{Z}$ can be expressed as:

$$
Z=U \Sigma U^{T}
$$

$\Lambda$ is a diagonal matrix, the elements on the diagonal are eigenvalues from large to small. $U$ is a feature vector according to the orthogonal array composed of columns. 


\subsubsection{STFT (Short-Time Fourier Transform)}

The time-frequency matrix str is acquired after short-time Fourier transform (STFT) of the sample signal. The signal time-frequency matrix describes the characteristic of amplitude distribution in a quite wide range from low frequency to high frequency comprehensively. Each element represents the amplitude on corresponding moment and corresponding frequency. It aims to get the number of singular value from large to small by singular value decomposition (SVD) [28] of the matrix str. A larger singular value contains the larger information of the matrix. SVD algorithm is described as follows:

$A_{m \times n}$ is the sample matrix and there are orthogonal matrix $U_{m \times n}$ and $V_{m \times n}$.

$$
A=U \Sigma V^{T}
$$

Among $S=\operatorname{diag}\left(\sigma_{1}, \ldots, \sigma_{r}\right), \sigma_{1} \geq \ldots \geq \sigma_{r}$ is the singular value of $A$.

\subsubsection{WT (Wavelet Transform)}

Firstly, we will decompose the signal with five-layer wavelet [29]. In this procedure, we could get the detailed and approximation coefficient. Then, we get the spectrum features of each coefficient-reconstructed signal by applying FFT. Correlation between mother wavelet and signal should be taken into consideration in wavelet decomposition. PIR signal shows strong symmetric feature, while at the same time high vanishing moment is required in noise reduction. Taking all these factors into account, we choose bior wavelet. It is symmetric which has a high vanishing moment and is regular.

\subsubsection{WPT (Wavelet Packet Transform)}

The WPT is similar to the WT, and the signal will be decomposed with five-layer wavelets for the wavelet packet coefficients [30]. Then, we can get the spectrum features of each coefficient-reconstructed signal by applying FFT to them. Also, the bior wavelet is also chosen to analyze the data.

\subsection{Feature Fusion and Recognition}

\subsubsection{FCEM (Fuzzy Comprehensive Evaluation Method)}

FCEM is based on the principle of the composition of fuzzy relation [31]. It is a method which can undertake a comprehensive evaluation from a number of factors for the membership degree condition of things. In this paper, the FCEM is used to judge the classification results from four sensors. Additionally, the recognition result can be obtained from the final data.

\subsubsection{SVM (Support Vector Machine)}

SVM is a classification method based on statistical learning theory [32]. It adopts principles of structural risk minimization and solves problems like limited samples, nonlinearity and high-dimensional pattern recognition to a large extent. Its main thought can be expounded as that no matter whether samples are linear and detachable or not, input sample vector can be mapped to a high-dimensional space. Then, optimal linear classification surface can be solved in the new space. A high-dimensional space adopts kernel function, as shown in following formula. 


$$
f(x)=\operatorname{sgn}\left(\sum_{i=1}^{l} a_{i} y_{i} K\left(x, x_{i}\right)+b\right)
$$

where $K\left(x, x_{i}\right)$ is a kernel function and $a_{i}$ is Lagrange multiplier. In this paper, $k$-fold cross validation is used to optimize classification results of SVM. Its basic idea is that original training data is divided into training data and test data, where $(k-1) n / k$ elements are selected from $n$ sample as the training data and the left $n / k$ acts as the test data to train SVM classifier, which is repeated for $M$ times. Then, we can obtain the recognition rate for an average of $M$ times, and get the final average recognition rate.

\section{Experiments and Results Analysis}

\subsection{Feature Extraction}

The number of test subjects is 10 . The walking paths are $\mathrm{A} 0(1,0)$ to $\mathrm{A} 1(1,6), \mathrm{B} 0(2,0)$ to $\mathrm{B} 1(2,6)$, $\mathrm{C} 0(3,0)$ to $\mathrm{C} 1(3,6), \mathrm{D} 0(4,0)$ to $\mathrm{D} 1(4,6), \mathrm{E} 0(5,0)$ to $\mathrm{E} 1(5,6)$, and $\mathrm{F} 0(6,0)$ to $\mathrm{F} 1(6,6)$. The test subjects were required to walk 10 times along each line, resulting in a total of 600 samples. Before feature extraction, the original data needs to be preprocessed, which includes waveform interception and noise cancelling. This paper adopts the method of noise cancelling. The sample is intercepted 300 points from the first peak of the waveform. The intercepted time-domain waveform of the test subject A and B are shown in Figure 9. In this figure, the abscissa represents time (s) and the ordinate represents the Voltage (V) value. And, each column represents different signal from 4 sensors (Row) for the same path. Each row represents different signal from the same sensor for 6 paths (Column), which can be seen in Figure 7.

Figure 9. The figure is the time domain waveform for target $A$ and $\operatorname{target} B$, where the blue solid line represents the target $\mathrm{A}$; the black dotted line represents the target $\mathrm{B}$; the abscissa represents time; the ordinate represents voltage; each column represents four sensors in different heights; each row represents six kinds of distances.

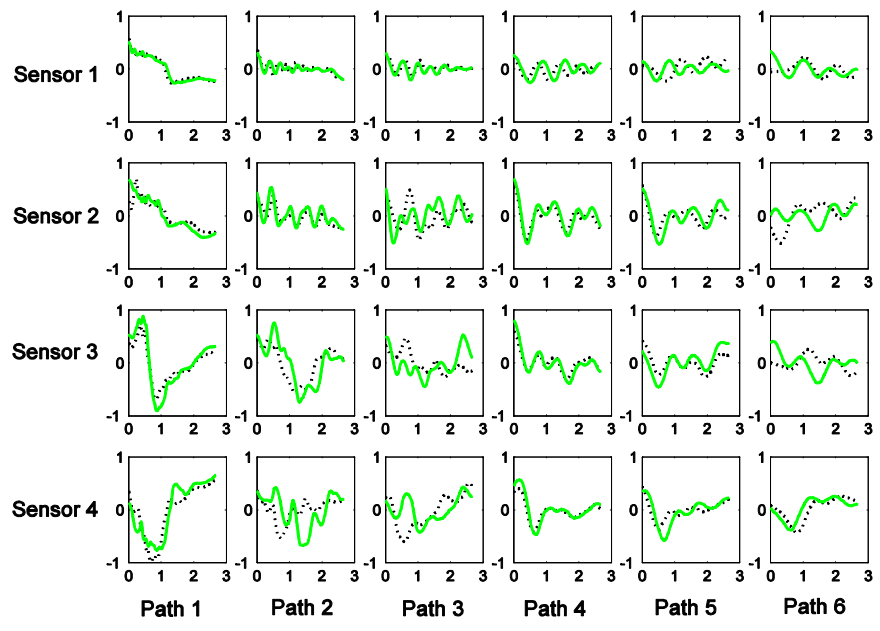

On one hand, the time-domain waveform of single target shows that there are some differences between the signal waveform from the same sensor node when the target walking in different paths. This is because of the changes in the relative position of the human target. With the increase of the distance, pyroelectric infrared sensor can get less and less human pyroelectric infrared signal which results in the decrease of amplitude. On the other hand, for the same distance target, there are significant differences 
in the observed signal waveform from different PIR nodes. The reason is that the PIR sensor module locates at different heights would detect the signal from different parts of human target body, which leads to the differences in the final waveform.

In the scenario of the same distance and PIR node, there is obvious difference between the observed signals from different targets caused by different characteristics of different targets.

As for the time domain signal, the four algorithms have been introduced in Section 5, which are used for feature extraction. The result of feature extraction of the two targets waveform is shown in Figure 10.

Figure 10a is feature extraction of FFT algorithm, the abscissa represents the frequency; the ordinate represents the amplitude; the blue solid line is spectrum feature of target A; the red dotted line is spectrum feature of target B.

Figure $10 \mathrm{~b}$ is feature extraction of STFT algorithm, the red line indicates that singular value distribution of time-frequency matrix of target $\mathrm{A}$; the green line indicates that singular value distribution of time-frequency matrix of target B.

Figure 10c is feature extraction of WT algorithm, the blue solid line indicates that WT energy distribution of target A; the red dotted line indicates that WT energy distribution of target B.

Figure $10 \mathrm{~d}$ is feature extraction of WPT algorithm; the blue solid line indicates that WPT energy distribution of target A, the red dotted line indicates that WPT energy distribution of target B.

Figure 10. Feature extraction by different algorithms. (a) Feature extraction by FFT.

(b) Feature extraction by STFT. (c) Feature extraction by WT. (d) Feature extraction by WPT.
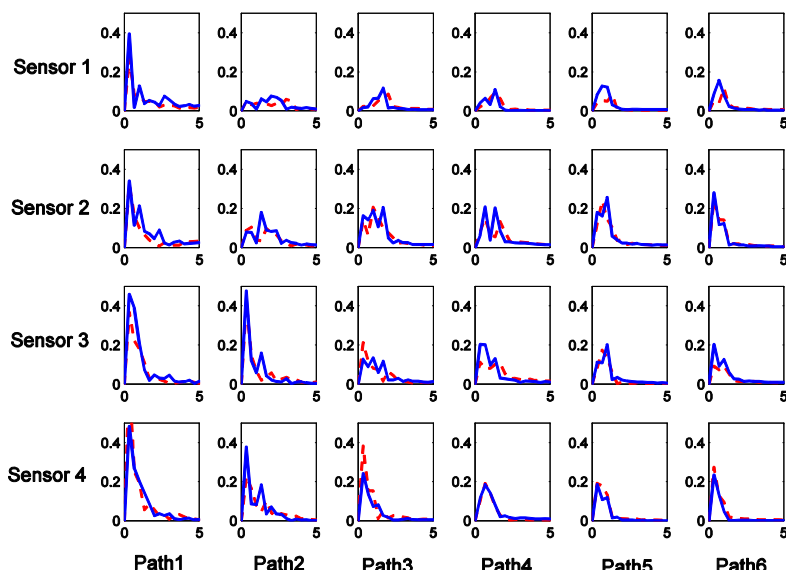

(a)
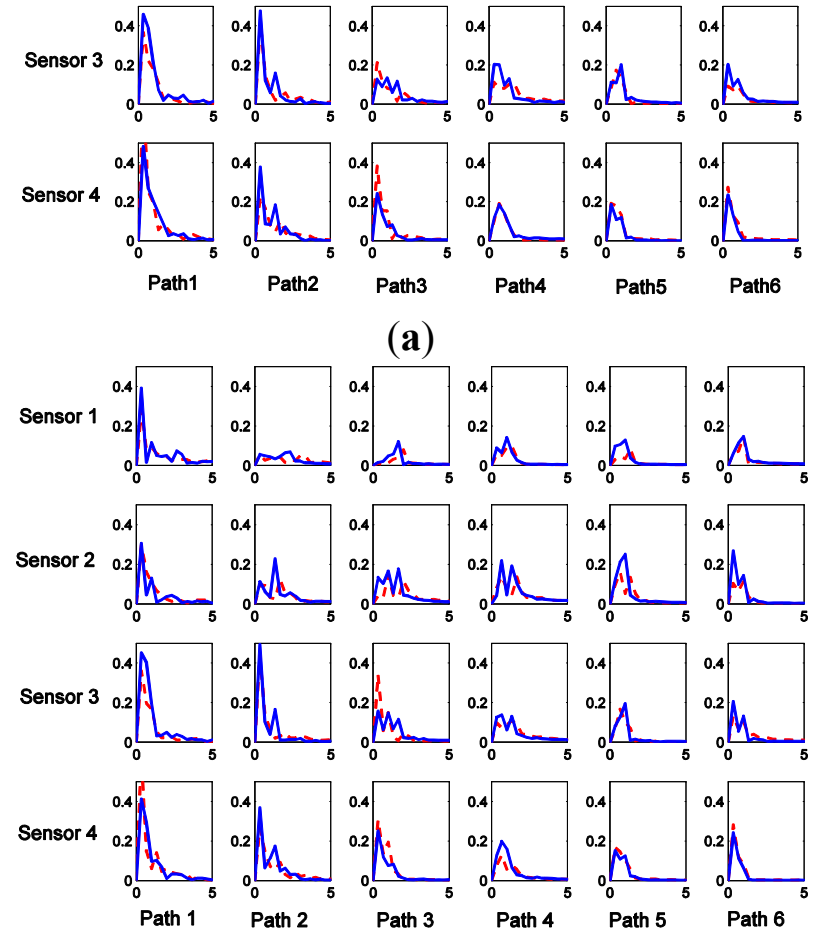

(c)
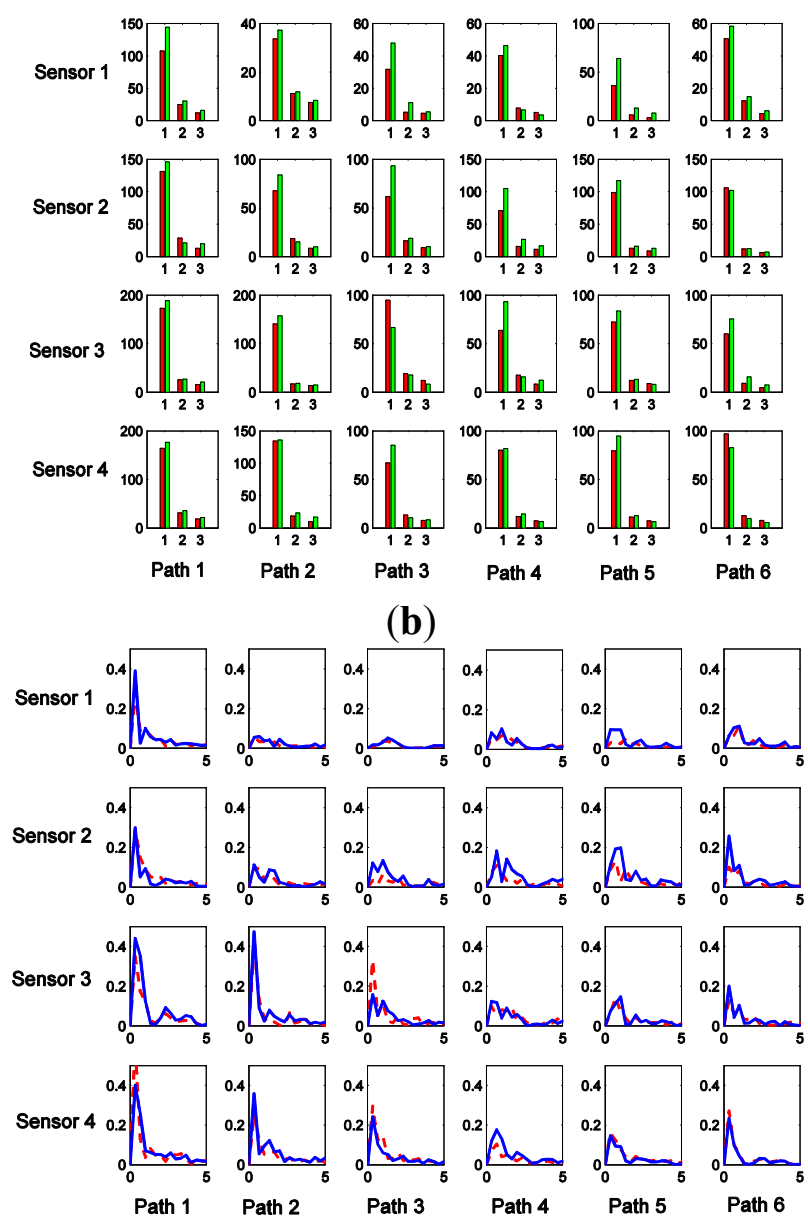

(d) 


\subsection{Comparison of Different Algorithms' Recognition Rates}

Figure 11 shows the recognition rate from the four PIR sensor modules under the independent operation. Also, the recognition rates are based on four different algorithms. As shown in Figure 11, the four PIR sensors are more sensitive to human target thermal infrared signal when targets are walking on the second path with a vertical distance of $2 \mathrm{~m}$ between sensor node and paths. Furthermore, it has the best result on recognition rate. Due to the changes in target walking path, there would be some changes in the observed waveform. Within a certain distance, as it increases, the PIR sensors get lots of weakened target radiation signals and become less sensitive to the detection of the target, which will cause the poor recognition results.

From the perspective of height, when the human target is walking, there would be different posture characteristics in different parts of the human body. So, for the same target, the sensors installed at different heights observe different kinds of characteristic signals. Moreover, due to the different distances, the signals will be also different even with the same height of sensor, thus directly influencing the discrimination of the sample data. These factors can affect the average recognition rate.

Figure 11. Recognition results under different algorithms. (a) Recognition results under FFT algorithm. (b) Recognition results under STFT algorithm. (c) Recognition results under WT algorithm. (d) Recognition results under WPT algorithm.

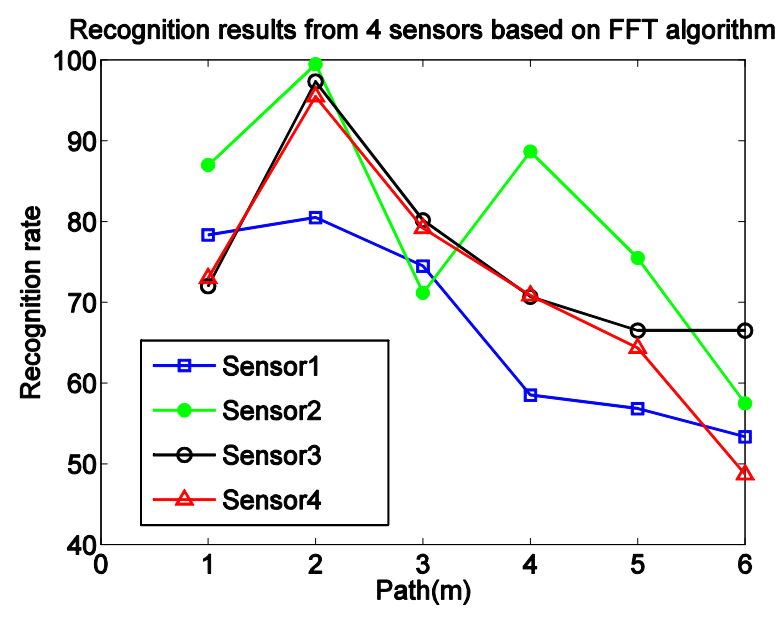

(a)

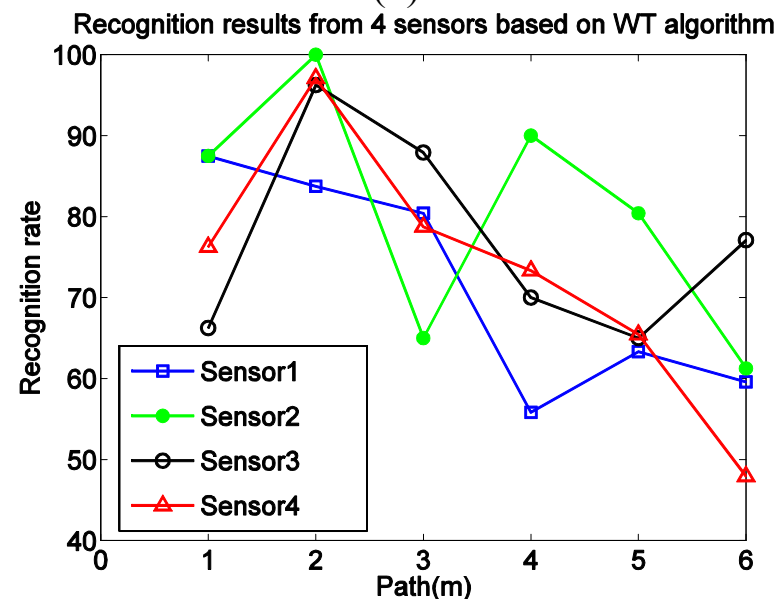

(c)

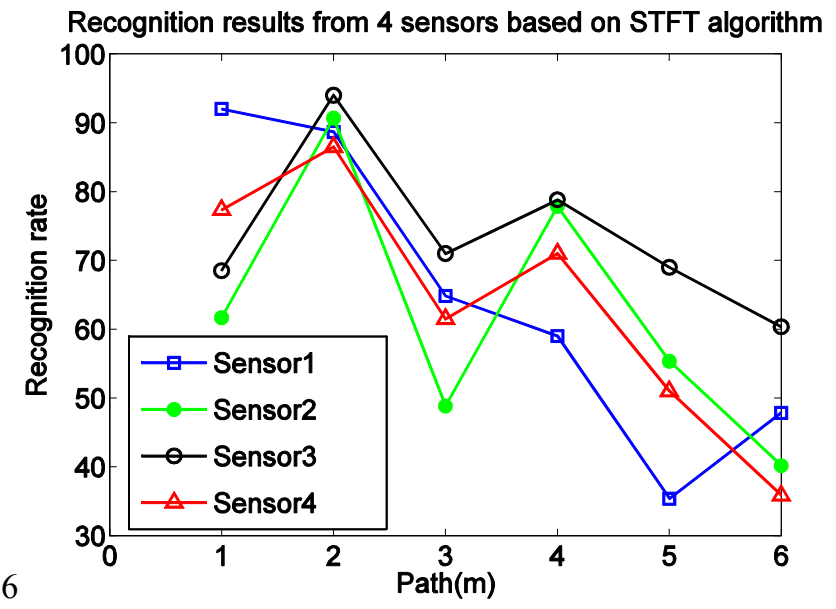

(b)

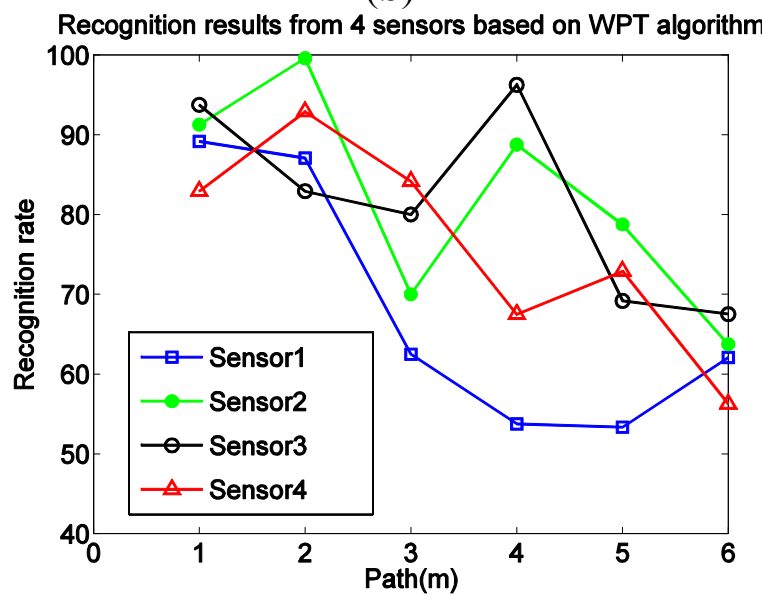

(d) 
When it comes to the issue that the recognition rate will decrease with the increase of the vertical distance between PIR sensor node and path, this paper proposes a solution based on decision level fusion. Figure 12 shows the comparison of recognition rate based on the different feature extraction algorithms and data fusion at the four PIR sensors. From Figure 12, it can be seen that the fusion schemes based on WT and WPT for feature extraction have relatively better recognition results.

Figure 13 reflects the upgrade percentage of recognition rate after fusion. It shows that the fusion system is more effective on human identification than that of the system without fusion procedure, especially for the scenario of remote target recognition. Table 3 shows the recognition rate and computing time based on different algorithms. It can be seen in the table that the average recognition rate of fusion programs based on WT and WPT for feature extraction are above $87.5 \%$ and $90.42 \%$, respectively. Experiments show that the fusion program, to some extent, can be used to solve the problem of decreasing recognition rate caused by the factor of distance.

From Figures 12 and 13, we can see that the WT feature is more suitable for realizing recognition, and thus can meet the basic requirements of human recognition.

Figure 12. The results of FCEM fusion recognition.

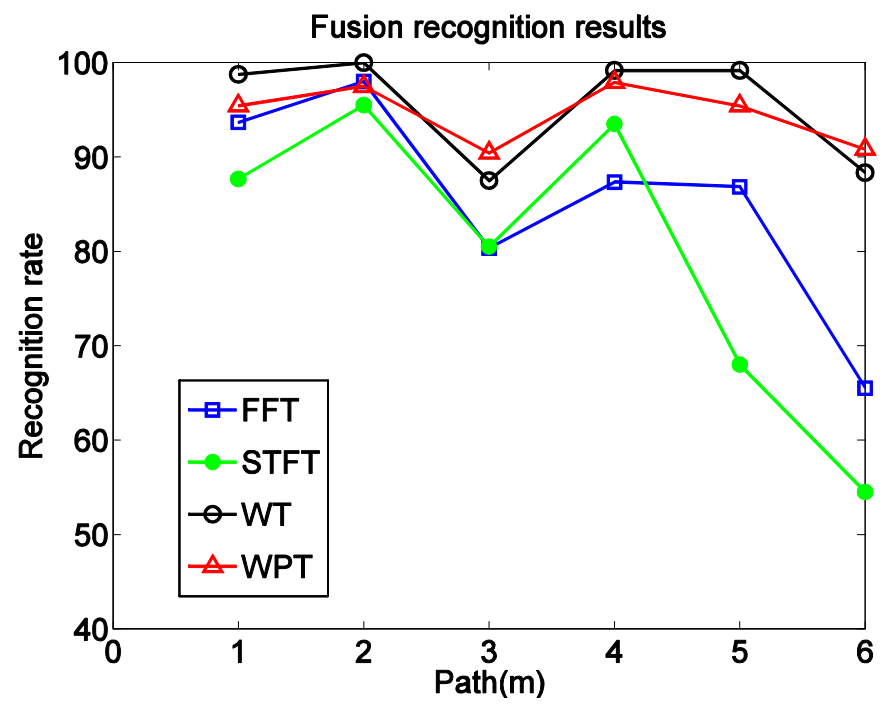

Figure 13. The upgrade percentage of recognition rate after fusion.

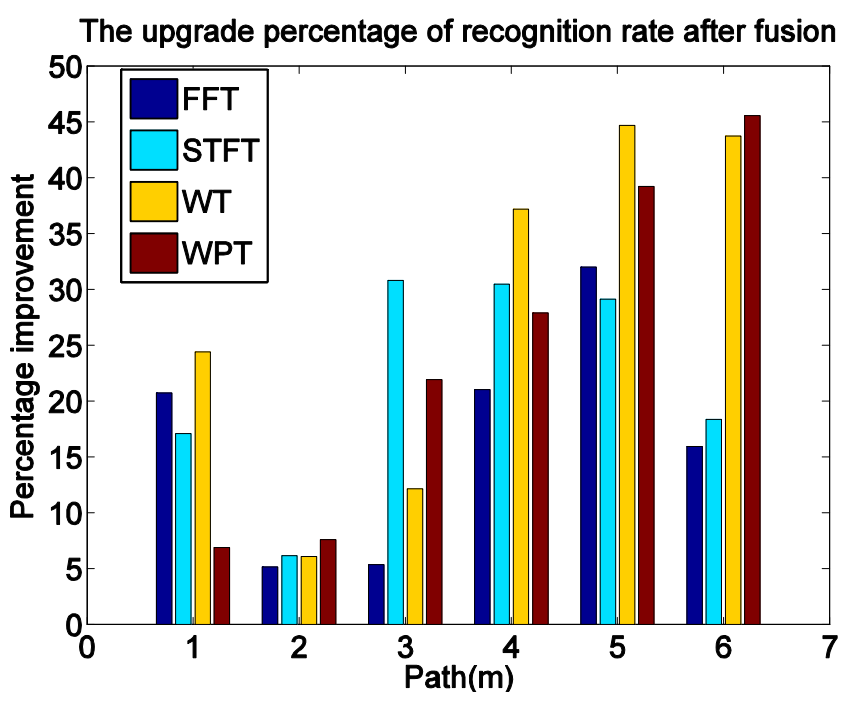


Table 3. Comparison of Recognition Results Based On Different Fusion Program.

\begin{tabular}{|c|c|c|c|c|c|c|c|}
\hline \multirow{2}{*}{ Algorithm } & \multicolumn{6}{|c|}{ Recognition rate in different path (\%) } & \multirow{2}{*}{ Computing time(s) } \\
\hline & Path1 & Path2 & Path3 & Path4 & Path5 & Path6 & \\
\hline $\mathrm{FFT}+\mathrm{PCA}+\mathrm{SVM}+\mathrm{FCE}$ & 83.5 & 89.6 & 76.3 & 86.0 & 77.7 & 65.0 & 10.1 \\
\hline $\mathrm{STFT}+\mathrm{SVM}+\mathrm{FCE}$ & 87.6 & 95.5 & 80.5 & 93.5 & 68.0 & 54.5 & 262.8 \\
\hline $\mathrm{WT}+\mathrm{SVM}+\mathrm{FCE}$ & 98.7 & 100 & 87.5 & 99.1 & 99.1 & 88.3 & 56.2 \\
\hline $\mathrm{WPT}+\mathrm{SVM}+\mathrm{FCE}$ & 95.4 & 97.5 & 90.4 & 97.9 & 95.4 & 90.8 & 25.0 \\
\hline
\end{tabular}

\section{Conclusions}

In this paper, as for the system with single PIR sensor, it shows through the comparison of recognition rate based on different feature extraction algorithms that with the increase of the vertical distance between PIR sensors and walking path, the recognition rate tends to decrease. To solve this problem, this paper proposes a solution to fuse the data from various sensors with different heights. Furthermore, extensive experiments show that the recognition results are improved when using the proposed fusion approach, which demonstrates that the proposed method can significantly reduce the false alarm rate of the existing PIR sensor and can provide a low-cost recognition solution for target identification.

\section{Acknowledgments}

This work is supported by National Natural Science Foundation of China with Grant No. 61170090.

\section{Author Contributions}

Fangmin Li proposed the idea of the paper; Wanchun Zhou conceived and designed the experiments; Ji Xiong, Na Jiang and Ning Zhao performed the experiments and analyzed the data; Wanchun Zhou wrote the paper.

\section{Conflicts of Interest}

The authors declare no conflict of interest.

\section{References}

1. Hong, L.; Ze, Y.; Zha, H.; Zou, Y.; Zhang, L. Robust human tracking based on multi-cue integration and mean-shift. Pattern Recognit. Lett. 2009, 30, 827-837.

2. Fan, J.; Xu, W.; Wu, Y.; Gong, Y. Human tracking using convolutional neural networks. IEEE Trans. Neural Netw. 2010, 21, 1610-1623.

3. Kang, S.J.; Samoilov, V.B.; Yoon, Y.S. Low-frequency response of pyroelectric sensors. IEEE Trans. Ultrason. Ferroelect. Freq. Contr. 1998, 45, 1255-1260.

4. Hussain, T.M.; Baig, A.M.; Saddawi, T.N.; Ahmed, S.A. Infrared pyroelectric sensor for detection of vehicular traffic using digital signal processing techniques. IEEE Trans. Veh. Technol. 1995, 44, 683-689.

5. Hossain, A.; Rashid, M.H. Pyroelectric detectors and their applications. IEEE Trans. Ind. Applic. 1991, 27, 824-829. 
6. Sekmen, A.S.; Wilkes, M.; Kawamura, K. An application of passive human-robot interaction: Human tracking based on attention distraction. IEEE Trans. Syst. Man Cybern. A 2002, 32, 248-259.

7. Hao, Q.; Brady, D.J.; Guenther, B.D.; Burchett, J.B.; Shankar, M.; Feller, S. Human tracking with wireless distributed pyroelectric sensors. IEEE Sens. J. 2006, 6, 1683-1696.

8. Moghavvemi, M.; Seng, L.C. Pyroelectric infrared sensor for intruder detection. In Proceedings of 2004 IEEE Region 10 Conference (TENCON 2004), Newark, NJ, USA, 21-24 November 2004; pp. 656-659.

9. Bai, Y.W.; Ku, Y.T. Automatic room light intensity detection and control using a microprocessor and light sensors. IEEE Trans. Consum. Electron. 2008, 54, 1173-1176.

10. Rajgarhia, A.; Stann, F.; Heidemann, J. Privacy-sensitive monitoring with a mix of IR sensors and cameras. In Proceedings of the Second International Workshop on Sensor and Actor Network Protocols and Applications, Boston, MA, USA, 22 August 2004; pp. 21-29.

11. Tao, S.; Kudo, M.; Nonaka, H.; Toyama, J. Person authentication and activities analysis in an office environment using a sensor network. Constr. Ambient Intell. 2012, 277, 119-127.

12. Bai, Y.-W.; Teng, H. Enhancement of the sensing distance of an embedded surveillance system with video streaming recording triggered by an infrared sensor circuit. In Proceedings of SICE Annual Conference, Tokyo, Japan, 20-2 August 2008; pp. 1657-1662.

13. Hao, Q.; Hu, F.; Xiao, Y. Multiple human tracking and identification with wireless distributed pyroelectric sensor systems. IEEE Sens. J. 2009, 3, 428-439.

14. Shankar, M.; Burchett, J.B.; Hao, Q.; Guenther, B.D.; Brady, D.J. Human-tracking systems using pyroelectric infrared detectors. Opt. Eng. 2006, 45, 106401.

15. Kim, H.H.; Ha, K.N.; Lee, S.; Lee, K.C. Resident location recognition algorithm using a Bayesian classifier in the PIR sensor-based indoor location-aware system. IEEE Trans. Syst. Man Cybern. C Appl. Rev. Arch. 2000, 39, 2410-2459.

16. Luo, X.; Shen, B.; Guo, X.; Luo, G.; Wang, G. Human tracking using ceiling pyroelectric infrared sensors. In Proceeding of 7th IEEE International Conference on Control and Automation, Christchurch, New Zealand, 9-11 December 2009; pp. 1716-1721, 2009.

17. Lu, J.; Gong, J.; Hao, Q.; Hu, F. Space encoding based compressive multiple human tracking with distributed binary pyroelectric infrared sensor networks. In Proceedings of IEEE Conference on Multiple Sensor Fusion and Integration, Hamburg, Germany, 13-15 September 2012; pp. 180-185.

18. Fang, J.-S.; Hao, Q.; Brady, D.J.; Shankar, M.; Guenther, B.D.; Pitsianis, N.P.; Hsu, K.Y. Path-dependent human identification using a pyroelectric infrared sensor and Fresnel lens arrays. Opt. Exp. 2006, 14, 609-624.

19. Fang, J.-S.; Hao, Q.; Brady, D.J.; Guenther, B.D.; Hsu, K.Y. Realtime human identification using a pyroelectric infrared detector array and hidden Markov models. Opt. Exp. 2006, 14, 6643-6658.

20. Zhou, X.; Hao, Q.; Fei, H. 1-bit walker recognition with distributed binary pyroelectric sensors. In Proceedings of 2010 IEEE Conference on Multisensor Fusion and Integration for Intelligent Systems, Salt Lake City, UT, USA, 5-7 September 2010; pp. 168-173.

21. Sun, Q.; Hu, F.; Hao, Q. Context Awareness Emergence for Distributed Binary Pyroelectric Sensors. In Proceedings of 2010 IEEE Conference on Multisensor Fusion and Integration for Intelligent Systems, Salt Lake City, UT, USA, 5-7 September 2010; pp. 162-167. 
22. Hu, F.; Sun, Q.; Hao, Q. Mobile Targets Region-of-Interest via Distributed Pyroelectric Sensor Network: Towards a Robust, Real-time Context Reasoning. In Proceedings of 2010 IEEE Conference on Sensors, Kona, HI, USA, 1-4 November 2010; pp. 1832-1836.

23. Sun, Q.; Hu, F.; Hao, Q. Mobile Target Scenario Recognition via Low-cost Pyroelectric Sensing System: Toward a Context-Enhanced Accurate Identification. IEEE Trans. on Syst. Man Cybern. Syst. 2014, 44, 375-384.

24. Sun, Q.; Lu, J.; Wu, Y.; Qiao, H.; Huang, X. Non-informative Hierarchical Bayesian Inference for Non-negative Matrix Factorization. Signal Process. 2015, 108, 309-321.

25. Chen, J.; Pi, D.; Liu, Z. An Insensitivity Fuzzy C-means Clustering Algorithm Based on Penalty Factor. J. Softw. 2013, 8, 2379-2384.

26. Su, X.; Jiang, W.; Xu, J.; Xu, P.; Deng, Y. A New Fuzzy Risk Analysis Method Based on Generalized Fuzzy Numbers. J. Softw. 2011, 6, 1755-1762.

27. Gumus, E.; Kilic, N.; Sertbas, A.; Ucan, O.N. Evaluation of face recognition techniques using PCA, wavelets and SVM. Expert Syst. Appl. 2010, 37, 6404-6408.

28. Jiang, Y.; Tang, B.; Qin, Y.; Liu, W. Feature extraction method of wind turbine based on adaptive Morlet wavelet and SVD. Renew. Energy 2011, 36, 2146-2153.

29. Kadambe, S.; Murray, R.; Boudreaux-Bartels, G.F. Wavelet transform-based QRS complex detector. IEEE Trans. Biomed. Eng. 1999, 46, 838-848.

30. Sun, Z.; Chang, C. Structural Damage Assessment Based on Wavelet Packet Transform. J. Struct. Eng. 2002, 128, 1354-1361.

31. Guo, L.; Gao, J.; Yang, J.; Kang, J. Criticality evaluation of petrochemical equipment based on fuzzy comprehensive evaluation and a BP neural network. J. Loss Prev. Process Ind. 2009, 22, 469-476.

32. Qian, H.; Mao, Y.; Xiang, W.; Wang, Z. Recognition of human activities using SVM multi-class classifier. Pattern Recognit. Lett. 2010, 31, 100-111.

(C) 2014 by the authors; licensee MDPI, Basel, Switzerland. This article is an open access article distributed under the terms and conditions of the Creative Commons Attribution license (http://creativecommons.org/licenses/by/4.0/). 